\title{
A new future for hip fracture care - orthogeriatrician lead in an 'Acute' Hip Unit
}

\author{
Authors: Abhaya Gupta ${ }^{A}$ and William Havelock ${ }^{B}$
}

\begin{abstract}
Although surgery is the definitive treatment for almost all hip fractures, there is evidence that outcomes such as morbidity and mortality are not determined by the type of surgery but by comorbidities and postoperative complications. A team approach, especially the involvement of an orthogeriatrician in managing medical issues, has been shown to improve surgical outcomes and should be encouraged in hospitals worldwide. An Acute Hip Unit is able to address the complex challenging needs of a frail older high-risk population soon after admission, therefore minimising delays. An orthogeriatrician-led unit can deal with the preoperative and complex postoperative medical, social, ethical, physical, and mental health issues that are associated with 'hip fracture syndrome', providing structured standardised evidence-based care by trained staff. This could be the model of care for the future.
\end{abstract}

KEYWORDS: Acute Hip Unit, hip fracture, orthogeriatrician

\section{Introduction}

Hip fractures pose a major public health burden and consume significant healthcare resources; the incidence of hip fractures is expected to rise with the ageing population and is already the second most frequent cause of hospitalisation in the older population. ${ }^{1}$ Hip fractures are associated with a significant increase in morbidity and mortality for both the short-term ( 3 to 6 months) and the long-term ( 5 to 10 years). ${ }^{2}$ A metaanalysis of prospective cohort studies found a five- to eight-fold increase in mortality rates within three months of fracture and estimated a one-year mortality range between $14 \%$ and $36 \% .^{3}$

Of those who survive one year after hip fracture, only $40 \%$ can perform all routine activities of daily living and only $54 \%$ can walk without an aid. ${ }^{4}$ Many patients require long-term nursing care, posing a challenge to both health and social care resources. The high impact of hip fracture probably results from a combination of trauma, major surgery, medical problems and low physiological reserve. Although surgery is the main treatment for a fracture of the hip, the orthogeriatrician

Authors: ${ }^{\text {A }}$ consultant geriatrician, Glangwili Hospital, Carmarthen, UK; ${ }^{\mathrm{B}}$ Consultant orthogeriatrician, University Hospital, Cardiff, UK has a key role in the further holistic management of the patient's medical comorbidities and complications during the perioperative period, and this specialist medical care can improve outcomes. In this article we present evidence indicating what care should be provided to those with hip fractures and how it should be delivered, and report how these findings have come together in the development of an Acute Hip Fracture Unit.

\section{Benefits of geriatrician-led perioperative medical management}

Patients with hip fractures have multiple complexities and are managed by several specialities, so there is a potential for miscommunication, delays and gaps in patient care. The National Institute of Clinical Excellence (NICE) in the UK has recommended that, beginning at admission, hip fracture patients should be offered a formal acute orthopaedic- or orthogeriatric-ward-based hip fracture programme, which includes orthogeriatric assessment. ${ }^{5}$ A formal Hip Fracture Programme, incorporating continuous orthogeriatric supervision and providing a co-ordinated structure and process of care, is more effective than a Geriatric Orthopaedic Rehabilitation Unit model or standard orthopaedic care. ${ }^{5}$ Geriatrician-led management for hip fracture patients can lead to significant reduction in both postoperative complications and mortality, ${ }^{6,7}$ and has also been shown to reduce length of stay in hospital. ${ }^{8,9}$

A study of a co-managed Hip Fracture Programme that began at admission showed that for every 1,000 patients treated in a geriatric model there were 17 fewer in-hospital deaths, 871 fewer complications and 97 fewer 30-day readmissions than was the case for 1,000 patients treated with standard care; this provides enormous financial benefits with the Hip Fracture Programme incurring only $66.7 \%$ of the expected costs. ${ }^{10}$

Where this care is delivered also has an impact on outcome: daily medical interventions were shown to reduce mortality whereas less-intensive interventions may not be as consistent in reducing medical complications. ${ }^{8}$ Focused multidisciplinary specialised care, covering the relatively homogenous hip fracture population within a defined geographic region, such as that which might be provided by an Acute Orthogeriatric Hip Unit, could improve outcomes, as has been the case for Stroke Units. ${ }^{11}$ 


\section{Preoperative optimisation to enable early surgery}

Reducing delays before surgery can reduce mortality and complications, as shown by a meta-analysis that found that earlier surgery is associated with a lower risk of death and with lower rates of postoperative pneumonia and pressure sores amongst older patients with hip fracture. ${ }^{12}$ Time to surgery can be reduced by the proactive identification and management of comorbid conditions and geriatric syndromes. Hip fractures occur mostly in the older population, with an average age at presentation of 84 years for men and 83 years for women; concurrent medical problems are common in this population. Unlike their younger contemporaries, older patients have a loss of functional reserve and a decline in organ function; it has been reported that $82 \%$ of patients have a significant medical condition that complicated a hip fracture. ${ }^{13}$ The altered physiological state, coupled with comorbidities, can be difficult to manage and increases perioperative risk.

Patients in classes 3 (severe systemic disease) and 4 (severe systemic disease that is a constant threat to life) of the American Society of Anaesthesiologists (ASA) Physical Status System have increased medical complications in the postoperative period, suggesting that these patients need to be closely managed medically, ${ }^{14}$ and any issues need to be addressed early in the preoperative stage.

Close liaison with the surgeon and anaesthetist about murmurs, cardiac findings and polypharmacy will help avoid routine tests that will not affect the surgical procedure. This, combined with the development of management protocols for common anticipated medical problems, will enable early surgery to take place. These findings support the importance of effective medical assessment and optimisation in the preoperative period.

\section{Postoperative complications: early identification and management}

Postoperative complications are common in hip fracture patients, amongst a population of more than 2,000 patients in a UK hospital followed prospectively, postoperative complications occurred in $20 \%$ of patients. Patients who had acute heart failure or postoperative chest infection had high 30-day mortality rates of $65 \%$ and $43 \%$, respectively. ${ }^{15}$ Monitoring, early identification and management of complications by the orthogeriatric team can improve outcomes for these frail older patients.

A number of common complications are often managed by the orthogeriatric team. A management strategy based on a comprehensive geriatric assessment ensures that multiple complex issues are managed efficiently and that interventions are tailored to individual patient needs.

\section{Delirium}

Delirium is commonly seen following hip fracture and occurs in up to $61 \%$ of patients, it is more common in patients with dementia. ${ }^{16}$ Delirium is a serious medical condition and increases the risk of poor outcomes, including surgical complications, mortality and institutionalisation. ${ }^{17}$ Delirium also delays functional recovery and hence affects the rehabilitation process. There are often multiple causes of delirium and many of the precipitating factors (Box 1) can be identified early so that treatment can be initiated proactively. There are many modifiable factors, such as management of electrolyte disturbances, treatment of infection, adequate analgesia and medication review. As noted in several studies, ${ }^{18-20}$ proactive orthogeriatric consultation can reduce the incidence and severity of delirium in hip fracture patients; nearly one-third of delirium incidences are completely preventable. ${ }^{17}$ In managing postoperative delirium, a holistic approach is needed as cause of the delirium is commonly multifactorial. Patients often require several targeted interventions, depending on their history and examination findings. The orthogeriatrician has an essential role in ensuring that serious medical conditions are not missed.

\section{Malnutrition}

About 23\% of patients with hip fractures are severely malnourished, and consequently can have muscle wasting, mental apathy and impaired cardiac function. Malnutrition results in impaired mobility and a tendency to postoperative complications and increased mortality. ${ }^{21}$

\section{Anaemia and inappropriate fluid status}

Anaemia is common in older people and is an independent risk factor for increased mortality, postoperative complications, poor functional outcome, and increased length of stay. ${ }^{22}$ Patients with symptomatic anaemia, especially those with known cardiac disease, require blood transfusion to keep haemoglobin levels at more than $80 \mathrm{~g} / \mathrm{l}^{23}$

\section{Problems with polypharmacy}

Polypharmacy is common in older patients whose medication regimes are often complex. An orthogeriatrician would routinely review medications for their dosage, indication, side effects and interactions.

\section{Dementia}

Dementia is noted in up to $19 \%$ of patients with hip fractures but cognitive impairment is found in up to $42 \%$ of patients. ${ }^{24}$ Cognitively impaired patients have longer length of hospital stay and higher physical dependence, mortality and institutionalisation. ${ }^{25}$ Assessment of cognitive status is complex

\section{Box 1. Precipitating factors for delirium.}

Electrolyte imbalance

Medications, especially opioids, sedatives, anticholinergics and antipsychotics

Metabolic disturbances

Dehydration

Alcohol withdrawal

Inadequate pain control

Drug toxicity

Urinary retention

Environmental changes 
if patients are in pain, and pain control for hip fracture patients may be suboptimal because of poor self-reporting of pain.

Cognitively impaired patients also benefit from comprehensive geriatric assessment and targeted intervention as a means of reducing delirium. ${ }^{26}$

\section{Bladder and bowel problems}

Urinary tract infection, urinary retention and incontinence are common postoperative problems following a hip fracture. Removal of urinary catheters the morning after surgery is likely to reduce the chances of urinary retention ${ }^{27}$ and also aids in early mobilisation. The diagnosis of constipation should be actively pursued and treated because this is more common in the postoperative period due to analgesia, immobility and dehydration.

\section{Pain}

Optimal pain control is essential so that patients can engage with rehabilitation. Patients with poorly controlled perioperative pain are likely to have increased length of stay and increased 6-month mortality. ${ }^{28}$ A Cochrane review has shown that nerve blocks can reduce the need for analgesic drugs and are effective for pain relief in hip fracture patients. ${ }^{29}$

\section{Other specialist roles of the orthogeriatrician working with hip fracture patients}

\section{Osteoporosis management}

The majority of hip fractures in the elderly are associated with osteoporosis, ${ }^{30}$ and following the fracture, risk factors for osteoporosis need to be addressed (Box 2). Orthogeriatricians have the expert knowledge and skills necessary for osteoporosis assessment and management, and this input from an orthogeriatrician can increase osteoporosis treatment in hip fracture patients from 12 to $69 \% .^{13}$

Several effective drugs are available for the management of osteoporosis and can reduce recurrent fractures (Box 3). Routine bone mineral density testing by dual energy X-ray absorptiometry (DEXA) is not recommended by NICE in the UK for female

\section{Box 2. Common risk factors for osteoporosis.}

Non-modifiable
$>$ Older age
$>$ History of previous fractures
$>$ History of maternal hip fracture
Modifiable
$>$ Prolonged use of steroids
$>$ Hyperthyroidism
$>$ Inactivity
$>$ Smoking
$>$ Excessive alcohol consumption
$>$ Multiple myeloma

\section{Box 3. Drugs for osteoporosis.}

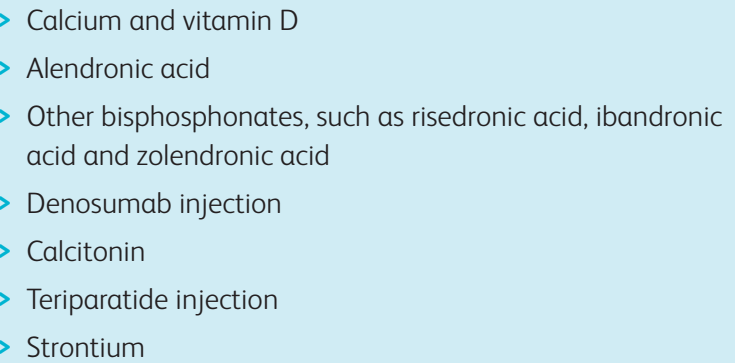

patients above the age of 75 years, ${ }^{5}$ but a clinical diagnosis can be made. Male patients are more likely to have secondary osteoporosis, whose associated and causative conditions would need to be addressed by an orthogeriatrician. Issues of recurrent fractures, atypical fracture, poor compliance, dental side effects, contraindications to bisphosphonate use, DEXA scan interpretation, and abnormal bone metabolism blood results can be addressed effectively by an orthogeriatrician.

\section{Assessment and management of falls}

A full falls assessment should be undertaken routinely by the orthogeriatrician. The circumstances leading to the fall need to be assessed with a detailed history and examination, which should include a history of syncope and factors contributing to the fall (Box 4). An orthogeriatrician would have links with the falls or syncope services available locally, ensuring timely and appropriate referrals.

\section{Rehabilitation}

A majority of patients do not return to pre-morbid activities of daily living after hip fracture. In one study, only $74 \%$ fully recovered walking ability and $56 \%$ returned to pre-fracture physical activities after one year; patients who were older and those with delirium after surgery recovered the least. ${ }^{31}$

Rehabilitation after hip fracture is essential to aid recovery and to prevent functional decline. Under the supervision of an orthogeriatrician, rehabilitation should begin on the first postoperative day. Early mobilisation should be encouraged to prevent chest infections, deep vein thrombosis and

\section{Box 4. Common risk factors for falls.}

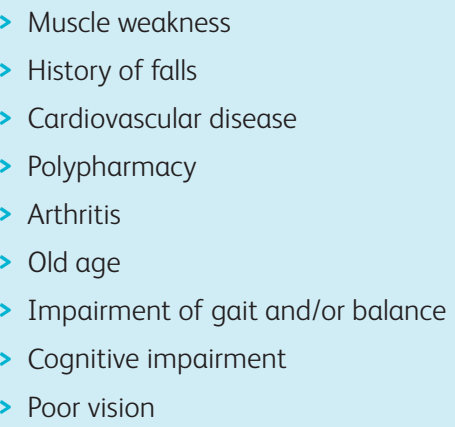


pressure sores. During discharge planning, a complex maze of cognitive, functional and social issues, the effects of acute and long-term medical comorbidities, the complexities of recovery from surgery, and the wishes of the patient and their family must be unravelled by an orthogeriatrician. The aims of rehabilitation should be to maximise potential within the hospital setting and then enable return to pre-admission residence by early supported discharge. Randomised trial data show that geriatric rehabilitation programs increase the likelihood of patients returning to the community. ${ }^{32}$ The relationship between rehabilitation program and functional gain in patients with cognitive impairment is complex. A systematic review showed beneficial effects of a rehabilitation programme on physical function in cognitively impaired patients, ${ }^{33}$ hence rehabilitation after hip fracture is mandatory even for cognitively impaired patients.

\section{Complex ethical issues}

Orthogeriatricians have a holistic approach to care for seriously ill patients and can advise on an appropriate approach towards end of life care, cardiopulmonary resuscitation, and the need for intensive care, coronary care, and ventilation. Depending on the patient's clinical condition, they can facilitate early decisions on complex issues. Early communication with the patient and their family is necessary, a process that is very familiar to the orthogeriatric team. A palliative-care approach, in liaison with the local palliative care team if needed, would

\section{Key points}

Hip fracture surgery is only one aspect amongst the multiple complexities of 'Hip fracture syndrome'.

Hip fractures are traditionally managed by surgeons but orthogeriatricians can play a key role in managing complex medical, social, and ethical conditions.

Prevention, early recognition and management of comorbidities and complications by orthogeriatric specialists are the key towards improving outcomes.

Orthogeriatricians can provide specialist input on falls prevention and osteoporosis management, and can lead multidisciplinary teams that support rehabilitation and recovery.

An orthogeriatrician-led Acute Hip Unit model can address several complex medical pre- and postoperative issues, providing standardised care in one ward even though surgical fixation is performed by different surgeons.

An Acute Hip Unit provides focussed, multidisciplinary, holistic, seamless and specialized early care by a trained staff, meeting national care standards.

An Acute Hip Unit care model is feasible across many UK hospitals. mean that patients and their families have minimal distress and receive adequate support.

\section{UK national standards approach}

Joint acute orthogeriatric co-management is recommended by $\mathrm{NICE}^{5}$ and by the British Orthopaedic Association and the British Geriatric Society. ${ }^{34}$ Shared surgical and medical care for hip fractures is increasingly being implemented and adopted across UK hospitals and worldwide. ${ }^{35}$ In the UK, a large audit database, The National Hip Fracture Database (NHFD), collects data about the organisation and processes involved in the pre- and postoperative care of hip fracture admissions across all hospitals in England and Wales; it has shown progressive improvements in hip fracture care. ${ }^{36}$ The latest NHFD report (2014) showed that $47 \%$ of hip fracture patients are assessed preoperatively by a geriatrician (up from $43 \%$ in 2013); 69\% received bone protection medication and $94 \%$ received falls assessment (up from 92\% in 2013). ${ }^{36}$

\section{First Acute Hip Unit in Wales}

The author (AG) developed an orthogeriatrician-led 15-bedded collaborative Acute Hip Unit in 2011 at Glangwili Hospital, Carmarthen, Wales; it was the first of its kind in Wales. ${ }^{37}$ The unit was modelled on Acute Coronary Care and Stroke Units, with dedicated ring-fenced beds, direct admission from the emergency department, standard protocols and care, staff training, and consistent senior medical cover. This unit has reduced length of stay and improved quality through the provision of standardised care. ${ }^{38}$ Fast-track protocols and the clerking of patients using a standardised clerking proforma in the Acute Hip Unit reduce the time spent in the emergency department, and allow data collection for audit and improvement purposes. A unit managed by just one geriatrician has led to the development of high-quality, structured, standardised, evidence-based care in managing the 'medical' issues of patients under the care of several surgeons, thereby reducing the chances of variable care and minimising preoperative delay.

Weekly multidisciplinary meetings led by a consultant geriatrician and increased daily medical supervision have reduced the units dependency on the on-call medical registrar, allowing sick patients to be managed consistently with earlier detection and management of delirium. This unit allows seamless preoperative and early postoperative care in the same environment.

Uniquely, in the new unit, care is shared between orthopaedic and medical juniors. This has improved training opportunities for orthopaedic juniors in assessing elderly patients' comorbidity, polypharmacy, falls and osteoporosis. The nursing staff also previously had an 'orthopaedic background' but now have a more specialised training and approach in dealing with both medical and surgical issues. Team working has improved considerably and improved morale and satisfaction is noted by the staff and patients. There is improved documentation of communication with patients, relatives and multidisciplinary professionals, and patients are provided with written information leaflets. Our experience shows that the social problems experienced by older hip fracture patients are the most difficult to tackle. This has been addressed by identifying 
problems proactively and by starting to plan possible solutions by coordinating with other professionals, agencies, the patient and their family; and by trying to minimise wastage of time and resources.

This model of care proves that cross-departmental working is achievable by reorganisation of existing resources available in an average district general hospital in the UK. With the rising demand for beds in acute hospitals in UK, this unit is an example of innovation in the care of hip fracture patients.

\section{Conclusions}

To reduce morbidity and mortality from hip fractures, attention must focus on optimising medical conditions preoperatively, on early surgery, and on preventing postoperative complications by early recognition and by providing specialist medical care throughout. During the perioperative period following a hip fracture, there are several areas of medical management that can be addressed optimally by an orthogeriatrician working together with the surgeon. Orthogeriatricians have specialist expertise in dealing with common medical issues, especially aspects of rehabilitation, falls recognition and prevention, secondary osteoporosis management and early recognition and management of delirium. Increasingly, and with good evidence to support them, orthogeriatric units are being set up in which care is shared between surgeons and an orthogeriatrician. An orthogeriatrician-led Acute Hip Unit model of care has many advantages and could feasibly be introduced into many hospitals by reorganisation of existing resources. This new care model has the potential to reduce morbidity and mortality in the frail hip fracture population.

\section{Acknowledgements}

We thank all of the staff of the Carmarthen Acute Hip Unit for their support in setting up the Unit.

\section{References}

1 Wilkins K. Health care consequences of falls for seniors. Health Rep 1999;10:47-55.

2 Wolinsky FD, Fitzgerald JF, Stump TE. The effect of hip fracture on mortality, hospitalization and functional status: a prospective study. Am J Public Health 1997;87:398-403.

3 Haentjens P, Magaziner J, Colón-Emeric CS et al. Meta-analysis: excess mortality after hip fracture among older women and men. Ann Intern Med 2010;152:380.

4 Zuckerman JD. Hip fracture. N Engl J Med 1996;334:1519-25.

5 National Institute for Health and Care Excellence, 2001. Hip fracture: the management of hip fracture in adults. Available online at www.nice.org.uk/guidance/CG124 [Accessed 6 August 2014].

6 Vidan M, Serra JA, Moreno C, Riquelme G, Ortiz J. Efficacy of a comprehensive geriatric intervention in older patients hospitalized for hip fracture: a randomized, controlled trial. J Am Geriatr Soc 2005;53:1476-82.

7 Fisher AA, Davis MW, Rubenach SE et al. Outcomes for older patients with hip fractures: the impact of orthopedic and geriatric medicine co-care. J Orthop Trauma 2006;20:172-8.

8 Antonelli Incalzi R, Gemma A, Capparella O et al. Continuous geriatric care in orthopaedic wards: a valuable alternative to orthogeriatric units. Aging (Milano) 1993;5:207-16.

9 Swanson CE, Day GA, Yelland CE et al. The management of elderly patients with femoral fractures: a randomised controlled trial of early intervention versus standard care. Med J Aust 1998;169:515-8.
10 Kates SL, Mendelson DA, Friedman SM. The value of an organized fracture program for the elderly: early results. J Orthop Trauma 2011;25:233-7.

11 Hill M. Hip fracture and stroke care: parallel problems in evidence letter. CMAJ 2002;167:845.

12 Simunovic N, Devereaux PJ, Sprague S et al. Effect of early surgery after hip fracture on mortality and complications: systematic review and meta-analysis. CMAJ 2010;182:1609-16.

13 Sainsbury R, Gillespie WJ, Armour PC, Newman EF. An orthopaedic geriatric rehabilitation unit: the first two years experience. $N Z \mathrm{Med} J$ 1986;99:583-5.

14 Donegan DJ, Gay N, Baldwin K et al. Use of medical comorbidities to predict complications after hip fracture surgery in the elderly. J Bone Joint Surg Am 2010;92:807-13.

15 Roche JJW, Wenn RT, Sahota O, Moran CG. Effects of comorbidities and postoperative complications on mortality after hip fractures in elderly people: prospective observational cohort study. $\mathrm{Br}$ Med J 2005;331:1374-6.

16 Robertson BJ, Robertson TJ. Postoperative delirium after hip fracture. J Bone J Surg 2006;88:2060-8.

17 Edelstein DM, Aharonoff GB, Karp A et al. Effect of postoperative delirium on outcome after hip fracture. Clin Orthop Relat Res 2004;422:195-200.

18 Siddiqui N, Holl R, Bitton AM, Holman J. Interventions for preventing delirium in hospitalised patients. Cochrane Database Syst Rev 2007; 18:CD005563.

19 Mercantonio ER, Flacker JM, Wright RJ, Resnick NM. Reducing delirium after hip fractures: a randomised trial. J Am Geriatr Soc 2001;49:516-22.

20 Lundström M, Olofsson B, Stenwall M et al. Postoperative delirium in old patients with femoral neck fractures: a randomised intervention study. Ageing Clin Exp Res 2007;19:178-86.

21 Koval KJ, Maurer SG, Su ET et al. The effects of nutritional status on outcomes after hip fractures. J Ortho Trauma 1999;13:164-9.

22 Dunne JR, Malone D, Tracey JK et al. Perioperative anaemia: an independent risk factor for mortality and resource utilisation in surgery. J Surg Res 2002;102:237-44.

23 Nelson AH, Fleisher LA, Rosenbaum SH. Relationship between postoperative anaemia and cardiac morbidity in high-risk vascular patients in the intensive care unit. Crit Care Med 1993;21:860-6.

24 Sietz DP, Adunuri N, Gill SS, Rochan PA. Prevalence of dementia and cognitive impairment among older adults with hip fractures. J Am Med Dir Assoc 2011;12:556-64.

25 Bentler SE, Liu L, Obrizan M et al. The aftermath of hip fracture: discharge placement, functional status change and mortality. Am J Epidemiol 2009;170:1290-9.

26 Nightingale S, Holmes J, Mason J, House A. Psychiatric illness and mortality after hip fractures. Lancet 2001;357:1264-5.

27 Michelson JD, Lotke PA, Steinberg ME. Urinary-bladder management after total joint-replacement surgery. N Engl J Med 1988;319:321-6.

28 Morrison RS, Magaziner J, McLaughlin MA et al. The impact of postoperative pain on outcomes following hip fracture. Pain 2003;103:303-11.

29 Parker MJ, Griffiths R, Appadu BN. Nerve blocks (subcostal, lateral cutaneous, femoral, triple, psoas) for hip fractures. Cochrane Database Syst Rev 2009;1:CD001159.

30 Shabat S. Hip fractures in elderly patients: perspectives towards the future. Disabil Rehabil 2005;27:1039-40.

31 Kagaya H, Shimada Y. Treatment and rehabilitation after hip fracture in the elderly. Crit Rev Phys Rehabil Med 2007;19:97-113.

32 Applegate M, Miller ST, Graney MJ et al. A randomised controlled trial of a geriatric assessment unit in a community rehabilitation hospital. N Eng J Med 1990;322:1572-8.

33 Muir SW, Yohannes A. The impact of cognitive impairment on rehabilitation outcomes in elderly patients admitted with a femoral neck fracture. A systematic review. J Geriatr Phys Ther 2009;32:24-32. 
34 British Orthopaedic Association, 2007. The care of patients with fragility fracture. Available online at www.nhfd.co.uk/20/hipfractureR.nsf/ welcome?readform [Accessed 6 August 2014].

35 Government of Western Australia, 2008. Orthogeriatric model of care. Available online at www.healthnetworks.health.wa.gov.au/modelsofcare/ docs/Orthogeriatric_Model_of_Care.pdf [Accessed 6 August 2014].

36 Royal College of Physicians. National Hip Fracture Database (NHFD). Available online at www.nhfd.co.uk/ [Accessed 6 August 2014].

37 Gupta A, Chenna S, Lorch A. A new collaborative acute hip unit improves care of osteoporosis and adherence to guidelines. Osteoporos Int 2012;23:S115.
38 Gupta A. The effectiveness of geriatrician led comprehensive hip fracture collaborative care in a new Acute Hip Unit based in a general hospital setting in the UK. J R Coll Physicians Edinb 2014;44:20-6.

Address for correspondence: Dr A Gupta, Glangwili Hospital, 6 Yddol Bronwydd Road, Carmarthen, Carmarthenshire, SA31 2AZ, UK.

Email: guptaabhaya@hotmail.com

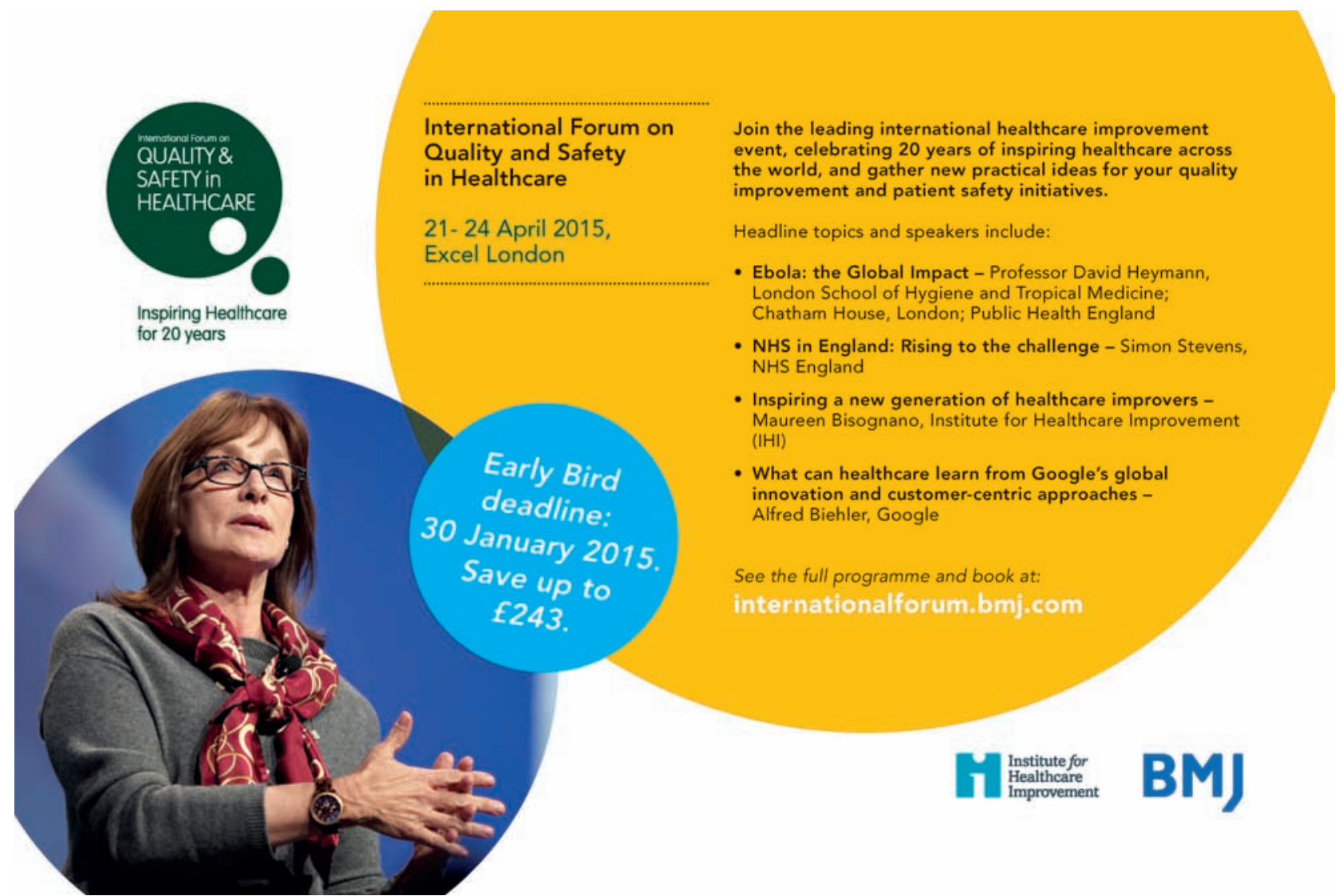

